\title{
MELIOIDOSIS IN BANGLADESH - A DISEASE YET TO BE EXPLORED !
}

Melioidosis, a potentially fatal infectious disease, is caused by Burkholderia pseudomallei (B.pseudomallei) which is a Gram negative organism usually present in the soil and stagnant surface water. Human is usually infected by traumatic inoculation of the organism from the soil, inhalation or by ingestion of contaminated food. ${ }^{1}$

Melioidosis is a disease of public health importance in Thailand, Vietnam, Malaysia, Laos, Myanmar and northern Australia where it is associated with high case-fatality rates. In endemic areas, seroepidemiological surveys have showed that the infection is fairly common in childhood as $80 \%$ of children had antibodies by the age of four years. ${ }^{2}$ In infected individuals the organism may remain dormant inside the phagocytic cells for months, years or decades. ${ }^{3}$ The factors that provoke reactivation of this latent, dormant "time bomb" are stress and alteration of immune status. Diabetes mellitus, chronic renal failure, cirrhosis of liver, AIDS, hematological malignancies seem to predispose the activation of the disease in an otherwise dormant latent infection. The manifestations are highly diverse ranging from acute pulmonary infection, septicemia, acute or chronic suppurative infections involving almost all organ systems to meningitis. ${ }^{4}$ Fulminant sepsis is much more common and is associated with high mortality.

Clinical diagnosis is often difficult, as the spectrum varies widely between benign and localized abscess to severe community acquired pneumonia to fatal septicemia. Many authors have rightly referred to this disease as "the remarkable imitator" and the "mimicker of maladies", since the disease can mimic, and produce similar symptoms caused by pyogenic bacterial infections, gram negative sepsis or tuberculosis. $^{5}$

B.pseudomallei is inherently resistant to a number of antibiotics and even with aggressive antibiotic therapy, the mortality rate remains high and the incidence of relapse is common. Mortality in disseminated septicemic melioidosis is $82-87 \%$. However, with ceftazidime therapy, the mortality rate was cut by half to $35-40 \%$. Fatalities are related to the speed of diagnosis and initiation of treatment. With prolonged maintenance treatment with cotrimoxazole relapse occurs in $4-23 \% .^{4}$
The organism possesses a number of virulence factors. The capsule and a type III secretion system of $B$. pseudomallei facilitates bacterial invasion of epithelial cells and intracellular survival. The presence of "exotoxin complex" is believed to be responsible for the fulminant course of a rapidly fatal septicaemia. ${ }^{3}$ Histopathology may show granuloma and necrosis mimicking caseation, suggestive of tuberculosis.

Melioidosis is rarely diagnosed in Bangladesh. In Bangladesh, the first case of melioidosis was reported in 1988 by ICDDR, B. ${ }^{6}$ Subsequently, between 1988 to 1999 five more cases were detected in United Kingdom among Bangladeshi immigrants from Sylhet region. ${ }^{7}$ Later on, in 2001 and in 2009 we have detected and reported 3 more cases of melioidosis among diabetic patients. All the three cases were from greater Mymensingh area. ${ }^{8-11}$

The presence of these cases strongly indicates that melioidosis is endemic in Bangladesh, particularly, in north eastern region of the country. As this disease is not familiar to most of the physicians and microbiologists, it is frequently misdiagnosed and microbiologists often ignore its growth in culture media as a laboratory contaminant. As such, the true incidence of the disease in Bangladesh may actually be much higher than is currently believed. Moreover, the climatic condition of Bangladesh is very favorable for survival of this organism and the disease is endemic in the neighboring countries like India and Myanmar. Villagers in Bangladesh live in close proximity to agricultural land and are likely to be exposed to this soil bacterium more frequently than anticipated. Therefore, systematic studies are required to determine the true magnitude and extent of this potentially fatal disease in Bangladesh. Also, physicians and medical microbiologists should be made aware to look for this important but yet unexplored infection.

\section{Md. Shariful Alam Jilani \\ Professor, Department of Microbiology \\ Ibrahim Medical College
J Ashraful Haq
Professor, Department of Microbiology
Ibrahim Medical College 


\section{Reference}

1. Raja NS, Ahmed MZ, Singh NN. Melioidosis: An emerging infectious disease. J Postgrad Med 2005; 51: 140-5.

2. Kanaphun P, Thirawattanasuk N, Suputtamongkol Y, et al. Serology and carriage of Pseudomonas pseudomallei: a prospective study in 1000 hospitalized children in noreast Thailand. J Infect Dis; 167: 230-3.

3. Jones AL, Bevendge TJ, Woods DE. Intracellular survival of Burkholderia pseudomallei. Infect Immun 1996; 64: 782-90.

4. Leelarasamee A. Burkholderia pseudomallei: the unbeatable foe? Southeast Asian J Trop Med Public Health 1998; 29(2): 410-5.

5. Loveleena, Chaudhry R, Dhawan B. Melioidosis: The remarkable imitator: Recent perspectives. J Assoc Physicians India 2004; 52: 417-20.
6. Streulenes MJ, Mondol G, et al. Melioidosis in Bangladesh - a case report. Trans $R$ S Trop Med Hyg 1988; 82: 778-79.

7. Kibblerl CC, Roberts CM, et al. Melioidosis in a patient from Bangladesh. Postgrad Med J 1991; 67: 764-766.

8. Halder D, Nik Zainal and Haq J Ashraful. Neonatal meningitis and septicaemia caused by Pseudomonas pseudomallei. Annals of Tropical Pediatrics 1998; 18: 161-164.

9. Nazimuddin K, Haq JA, et al. Melioidosis - a case report. J Bang Coll Phy Sur 2001; 19: 71-74.

10. Haq JA. Melioidosis in Bangladesh. Presented in Scientific Seminar, Mymensingh Medical College 2009; 10.

11. Haq J Ashraful. Melioidosis in Bangladesh - To See or Not to See. Bangladesh Journal of Pathology 1998; 13(1): 1-2. 